Edición Extra-Ordinaria. ISSN 2027-1034 P.p 666 - 675

Memorias del VII Encuentro Nacional de Experiencias en la Enseñanza de la Biología y la Educación Ambiental y II Congreso Nacional de Investigación en la Enseñanza de la Biología

\title{
APROXIMACIÓN A LAS TENDENCIAS INVESTIGATIVAS SOBRE LA ENSEÑANZA DE LA TEORÍA EVOLUTIVA EN RELACIÓN CON EL CONTEXTO SOCIAL, CULTURAL Y POLÍTICO ${ }^{1}$
}

\section{APPROACH TO THE RESEARCH TRENDS ON THE TEACHING OF EVOLUTIONARY THEORY IN RELATION TO THE SOCIAL, CULTURAL AND POLITICAL CONTEXT}

\author{
Gonzalo Peñaloza Jiménez ${ }^{2}$ \\ Carlos Javier Mosquera Suárez ${ }^{3}$
}

\section{Resumen}

La teoría de la evolución biológica provoca debates de tipo religioso, ético, político y educativo. Por tal razón, la investigación sobre su enseñanza es muy amplia y diversa. En este texto se presenta una aproximación a las principales tendencias investigativas sobre la enseñanza y aprendizaje de la evolución que consideran aspectos sociales, culturales y políticos. Al final se esbozan algunos apuntes metodológicos sobre la investigación en la enseñanza y el aprendizaje de la evolución. Este trabajo es resultado de una revisión bibliográfica conducente a la formulación de un problema de tesis doctoral.

Palabras Clave: evolución biológica, enseñanza, creencias, contexto.

\section{Abstract}

The theory of biological evolution causes religious, ethical, political and educational debates. For this reason, research on its teaching is vast and diverse. This paper presents an approach to the main research trends on the teaching and learning of evolution which consider social, cultural and political issues. At the end, outlines some methodological notes about research on teaching and learning of evolution. This work results from a literature review leading to the formulation of a doctoral thesis problem.

Key Words: biological evolution, teaching, beliefs, context.

\footnotetext{
1 Esta ponencia es parte de los avances de la tesis de doctorado del primer autor bajo dirección del segundo. Se realiza en el marco de la beca otorgada por Colciencias en la Convocatoria nacional para estudios de doctorado en Colombia - 528 de 2011.

2 Estudiante del Doctorado Interinstitucional en Educación Universidad Distrital Francisco José de Caldas Bogotá - Colombia.

3 Profesor del Doctorado Interinstitucional en Educación Universidad Distrital Francisco José de Caldas Bogotá - Colombia.
} 
Edición Extra-Ordinaria. ISSN 2027-1034 P.p 666 - 675

Memorias del VII Encuentro Nacional de Experiencias en la Enseñanza de la Biologia y la Educación Ambiental y II Congreso Nacional de Investigación en la Enseñanza de la Biología

\section{Introducción}

La teoría de la evolución es la teoría central y unificadora de la biología debido a su capacidad explicativa de la diversidad de la vida y su sustento fáctico y conceptual (Gould, 1987; Mayr, 2000; Pigliucci, 2007). No obstante, la aceptación y la comprensión de sus fundamentos por parte del público, es muy baja (Miller, Scott y Okamoto, 2006). Frente a la evolución biológica, las personas - colectiva o individualmente - adoptan posturas de rechazo, aceptación o sincretismo (Munger, 2002).

En el campo educativo la controversia -entre quienes aceptan o rechazan la evolución- es expresa y toma matices en cada país y período. En muchos países la enseñanza de la evolución está contenida en los estándares educativos, mientras que en otros no lo está e incluso se proscribe.

\section{Estado Actual De La Investigación}

Las investigaciones sobre la enseñanza y el aprendizaje de la evolución biológica (EB) han analizado, de una parte aspectos didácticos, cognitivos, conceptuales, curriculares, de formación del profesorado; y de otro lado, han abordado el papel del contexto sociopolítico en diferentes aspectos de la enseñanza de la evolución. Esta aproximación se ocupa de aquellos análisis que consideran factores sociales, culturales y políticos en relación con la enseñanza de la Teoría.

\section{Estudios sobre los factores sociales, culturales y políticos relacionados con la enseñanza y aprendizaje de la teoría de la evolución biológica}

La mayoría de las investigaciones educativas en torno a la EB ponen de relieve la necesidad de considerar el contexto cultural, social y político -especialmente las creencias religiosas-, como factores que influyen directamente en la enseñanza y el aprendizaje de dicha teoría. Los estudios muestran que debe tenerse en cuenta que la evolución suscita una controversia social en la que intervienen intereses ideológicos, políticos y religiosos (Clément, 2004; Hernández, 2002) y que dicho debate desemboca, generalmente, en un conflicto entre ciencia y religión (Shankar, 1990).

Desde el punto de vista educativo esta condición "polémica", tiene una gran riqueza pedagógica y didáctica puesto que implica abordar cuestiones como: la naturaleza de la conocimiento científico, la epistemología, la relación ciencia y cultura, la influencia de la religión en la educación, entre otras.

A continuación se agrupan los estudios que de una u otra manera se han ocupado de factores conexos, y en algunos casos determinantes, para la enseñanza y 
Edición Extra-Ordinaria. ISSN 2027-1034 P. p 666 - 675

Memorias del VII Encuentro Nacional de Experiencias en la Enseñanza de la Biologia y la Educación Ambiental y II Congreso Nacional de Investigación en la Enseñanza de la Biología

aprendizaje de la TEB. Se detallan los estudios que se consideran más relevantes.

Análisis del currículo de biología con respecto a la enseñanza de la evolución y el creacionismo (Donnelly y Boone, 2007; Skoog, 1978; Fail, 2008). Se analiza la importancia dada a la EB en los currículos de ciencias en educación primaria y secundaria. Indagan sobre la posibilidad de incluir la evolución en todos los grados escolares. El estudio de Borczyk (2010) llama la atención sobre las consecuencias conceptuales y didácticas de conminarla a un grado en particular.

Aspectos legales de la controversia entre enseñanza de la evolución y el creacionismo (Moore y Cotner, 2009; Skoog, 1979). Estos estudios se han desarrollado particularmente en Estados Unidos en donde se han dado disputas legales en torno a la enseñanza de la evolución biológica en las escuelas públicas. Se detallan los "juicios" y sus decisiones, los cuales evidencian las posturas de grupos religiosos y políticos, así como de la comunidad científica.

Análisis de los libros de texto de biología para determinar posturas antievolucionistas y errores en la enseñanza de la evolución (Carlesen, 1991; Hellman, 1965; Hernández, 1994; Jiménez, 1994; Rosenthal, 1985; Skoog, 1979, 1984).

Relación entre la formación de los maestros, su comprensión y enseñanza de la teoría evolutiva. (Campos, Alucema y Barahona, 2000; Campos, Cortés y Rossi, 2002; Cornett, Yeotis, y Terwilliger, 1990; Gregory, 2009; Jiménez, 1991; Maciel, 2007; Moore y Cotner, 2009a, 2009b; Nadelson y Southerland, 2010; PazMiño y Espinosa, 2009; Roelfs, 1987; Shankar, 1990). Estas investigaciones se concentran en la influencia que tiene la formación académica disciplinar en la enseñanza de la evolución en las aulas. En tal sentido, por ejemplo, Shankar (2010) afirma que el 28\% de los profesores de biología del Estado de Texas (EU) enseñan creacionismo y la formación de profesores inadecuada es una de las variables asociadas con ello.

Los estudios de Aguillard (1998) y Aguillard y Aguillard (1999) en Louisiana (EU) indagan por la formación en evolución biológica en maestros de ciencias. Concluyen que hay una correlación entre la formación del profesorado y el énfasis que ellos dan a la evolución en sus cursos de biología. En el mismo sentido, Roelf (1987) en EU encontró que la formación de profesores incide positivamente en el énfasis y rigurosidad dada a la EB en las aulas. Maciel (2005) tras analizar las concepciones de estudiantes mexicanos de licenciatura en educación primaria, concluye que existen concepciones erróneas predominantes sobre la EB.

Influencia del conservadurismo religioso (Aguillard, 1998; Aguilard y Aguillard, 1999; Bates, 1976; Berkman, Sandell y Plutzer, 2008; Clément y otros, 2008; Ellis, 
Edición Extra-Ordinaria. ISSN 2027-1034 P.p 666 - 675

Memorias del VII Encuentro Nacional de Experiencias en la Enseñanza de la Biologia y la Educación Ambiental y II Congreso Nacional de Investigación en la Enseñanza de La Biología

1983; Maldonado-Rivera, 1998; Peker, Gul y Aykut, 2010; Southcott y Downie, 2012; Shankar, 1990; Soto-sonera, 2006, 2009; Zimmerman, 1987). Las investigaciones acerca del papel de las creencias religiosas de los profesores y estudiantes han encontrado, que a pesar de que la EB haga parte del currículo y su enseñanza oficialmente no se proscriba, en el aula los procesos de enseñanza y aprendizaje se limitan, tergiversan o suprimen por las posiciones ideológicas y religiosas de quienes hacen parte de la comunidad educativa o de las entidades encargadas de trazar o implementar las políticas educativas.

En un estudio realizado en Túnez, se reconoce la dificultad de los estudiantes para diferenciar entre el razonamiento religioso y biológico y sus problemas para comprender el estatus científico de la EB, debido a la limitada comprensión de naturaleza de la ciencia (Aroua, Coquide y Abbes, 2009: 475).

BouJaoude y otros (2011a) analizaron las concepciones sobre la evolución biológica entre estudiantes de secundaria musulmanes de Egipto y Líbano. Se estableció que los estudiantes sostienen concepciones erradas sobre la evolución y la ciencia. La incomprensión de ciertos aspectos de la EB tienen relación con sus creencias religiosas.

En un estudio similar BouJaoude y otros (2011b) analizaron las ideas de profesores de secundaria del Líbano. Concluyen que a pesar de los años de formación y enseñanza de la biología, algunos maestros no modifican sus ideas con respecto a la evolución, debido a sus creencias religiosas. Es decir, si bien la formación disciplinar puede alterar la aceptación de la EB, del conocimiento no se deriva la creencia.

En Turquía, Özay (2010) encuestó a profesores y estudiantes encontrando que la mayoría rechaza la evolución y esto se relaciona con sus creencias religiosas. Los encuestados creen que hay una dicotomía entre sus creencias religiosas y la aceptación de la evolución, consideran que si aceptan una, deben rechazar la otra. El autor llama la atención sobre la diferencia entre comprensión y creencia, enfatizando que ellas se ocupan de cuestiones de diferente naturaleza, mientras una se ocupa de las causas naturales y los fenómenos naturales, la otra se ocupa de la creencia, el significado y el propósito (Özay, 2010:197).

Clément y otros (2008) realizaron un estudio en 14 países (5 de África, 1 del Medio Oriente y 8 de Europa) entre profesores de ciencias naturales encontrando un alto grado de correlación entre la creencia en dios, el grado de práctica de la religión, las creencias creacionistas y la aceptación o rechazo de la evolución biológica.

En Polonia Borczyk (2010) discute sobre los cambios políticos y sociales que han 
Edición Extra-Ordinaria. ISSN 2027-1034 P.p 666 - 675

Memorias del VII Encuentro Nacional de Experiencias en la Enseñanza de la Biología y la Educación Ambiental y II Congreso Nacional de Investigación en la Enseñanza de la Biología

posibilitado la emergencia de grupos creacionistas y la forma en que tienen creciente influencia en la sociedad polaca y la educación.

Paz-Miño y Espinosa (2011) encuestaron profesores universitarios de especialidades biológicas y no biológicas, de instituciones religiosas y seculares privadas o públicas. Además estudiantes de diferentes niveles. En total se encuestaron 244 docentes de 35 instituciones. El estudio concluye que la formación educativa y las creencias religiosas son factores relacionados con la aceptación de la evolución biológica. Se encuentra diferencias en la aceptación entre instituciones religiosas y seculares.

En Puerto Rico, Soto-Sonera (2009) realizó un estudio de caso en el que se analiza el papel de las creencias religiosas de los docentes en las posturas sobre la EB y su didáctica. El autor concluye que: “... la postura o versión hegemónica que asume el docente en relación con esta explicación científica [TEB] se vincula con el valor que otorgan a las creencias religiosas que profesan."

El-Hani y Sepulveda (2010) investigan sobre la relación entre ciencia y religión en la educación de profesores en formación de ciencias. Mediante el análisis de las concepciones de la naturaleza de los maestros se establece la relación entre las visiones religiosas y científicas.

\section{Conclusiones}

En términos generales las diferentes investigaciones analizadas coinciden en señalar la importancia del contexto social, cultural y político en la enseñanza y aprendizaje de la evolución biológica. Se denota en ellas, que las creencias religiosas, la formación disciplinar de los maestros, las circunstancias políticas de cada país, la comunidad, entre otros factores, juegan un importante papel en los procesos educativos. Se señala que elementos como el currículo, los libros de texto, la disposición y las actitudes del maestro para enseñar la evolución biológica, son incididos por el ambiente político y cultural de un país o comunidad.

Las consideraciones anteriores muestran un amplio e interesante campo de investigación para la didáctica de las ciencias en la medida en que en la enseñanza de la evolución biológica se conjugan elementos como ciencia, cultura, creencias, conocimiento, entre otros. Al abordar esta cuestión se hace patente la necesidad de considerar las dimensiones sociales, culturales y políticas asociadas con la enseñanza y el aprendizaje de la evolución biológica y no solo establecer medios, estrategias y maneras para "enseñar mejor" centrándose exclusivamente en los contenidos. Es decir, se requiere analizar la enseñanza y el aprendizaje en relación con los factores que configuran el contexto en el que realmente se enseña y se aprende la evolución biológica. 
Bio-grafia Escritos sobre la Biologia y su Enseñanza.

Edición Extra-Ordinaria. ISSN 2027-1034 P.p 666 - 675

Memorias del VII Encuentro Nacional de Experiencias en la Enseñanza de la Biología y la Educación Ambiental y II Congreso Nacional de Investigación en la Enseñanza de la Biología

\section{Bibliografía}

Aguillard, E. y Aguillard, D. (1999). Evolution Education in Louisiana Public Schools: A Decade Following. The American Biology Teacher, 61(3), 182-188

Aguillard, D. (1998). An analysis of factors influencing the teaching of biological evolution in Louisiana public secondary schools. (Tesis doctoral). Louisiana State University and Agricultural \& Mechanical College, EU.

Aroua, S., Coquide, M. \& Abbes, S. (2009). Overcoming the Effect of the Sociocultural Context: Impact of Teaching Evolution in Tunisia. Evo Edu Outreach 2, 474-478

Bates, V. L. (1976). Christian Fundamentalism and the Theory of Evolution in Public Schools: A Study of the Creation Science Movement. (Tesis doctoral). University of California, Davis, EU.

Berkman, B., Sandell, J. y Plutzer, E. (2008). Evolution and Creationism in America's Classrooms: A National Portrait. PLoS Biology, 6 (5): 920-924.

Borczyk, B. (2010). Creationism and the Teaching of Evolution in Poland. Evo Edu Outreach, 3, 614-620.

BouJaoude, S., Wiles, J., Asghar, A. y Alters, B. (2011a). Muslim Egyptian and Lebanese Students' Conceptions of Biological Evolution. Science \& Education, 20: 895-915.

BouJaoude, S., Wiles, J., Asghar, A., Jaber L., Sarieddine, D. y Alters, B. (2011b). Biology professors' and teachers' positions regarding biological evolution and evolution education in a Middle Eastern Society. International Journal of Science Education, 33(7), 979-1000.

Campos, M. A., Alucema, M. A. y Barahona, A. (2000). Análisis lógicoepistemológico de la conceptualización de teoría evolutiva moderna de estudiantes de licenciatura. Memoria electrónica del área II: currículum, procesos y prácticas educativas. V Congreso Nacional de Investigación Educativa. Aguascalientes, México.

Campos, M. A., Cortés, L. y Rossi, A. (2002). Dinámica de la construcción de conocimiento científico sobre la teoría sintética de la evolución en el aula universitaria. Revista Intercontinental de Psicología y Educación, 4(2), 21-33. 
Bio-grafia Escritos sobre la Biologia y su Enseñanza.

Edición Extra-Ordinaria. ISSN 2027-1034 P. p 666 - 675

Memorias del VII Encuentro Nacional de Experiencias en la Enseñanza de la Biología y la Educación Ambiental y II Congreso Nacional de Investigación en la Enseñanza de la Biología

Carlesen, W. S. (1991). Effects of new biology teachers' subject-matter knowledge on curricular planning. Science Education 75, 631-647.

Clément, P. (2004). Science et idéologie: exemples en didactique et épistémologie de la biologie. Actas del Colloque Sciences, médias e société, 53-69.

Clément, P., Quesada, M.P., Laurrent, C. y Carvalho, G. (2008). Science and Religion: Evolutionism and Creationism in Education. A survey of teachers conceptions in 14 countries. XIII IOSTE Symposium, The use of Science and Technology Education for Peace and Sustainable Development.

Cornett, J. W., Yeotis, C. and Terwilliger, L. (1990), Teacher personal practical theories and their influence upon teacher curricular and instructional actions: $A$ case study of a secondary science teacher. Science Education, 74, 517-529.

Donnelly, L. y Boone, W. (2007). Biology teachers' attitudes toward and use of Indiana's evolution standards. Journal of Research in Science Teaching, 44(2), 236-257

El-Hani, C. y Sepulveda, C. (2010). The relationship between science and religion in the education of protestant biology preservice teachers in a Brazilian university. Cult Stud of Sci Educ 5, 103-125

Ellis, W. E. (1983). Biology teachers and border state beliefs. Transactions: Social Science and Modern Society, 20, 126-130.

Gould, S. J. (1987). Darwinism Defined: The Difference Between Fact and Theory. Discover, 8(1), 64-70.

Gregory, T. R. (2009). Understanding Natural Selection: Essential Concepts and Common Misconceptions. Evo Edu Outreach, 2(2), 156-175

Hellman, R. A. (1965). Evolution in American School Biology Books from the late Nineteenth Century Until the 1930s. American Biology Teacher, 27: 778-780.

Hernández, M. (1994). El papel del conocimiento previo y la legibilidad del libro de texto en el aprendizaje de la teoría sintética de la evolución en la escuela secundaria, (Tesis de maestría). Facultad de Ciencias, UNAM, México.

Hernández, M. (2002). La historia de la enseñanza de la teoría de la selección natural. (Tesis doctoral). UNAM, México. 
Edición Extra-Ordinaria. ISSN 2027-1034 P.p 666 - 675

Memorias del VII Encuentro Nacional de Experiencias en la Enseñanza de la Biologia y la Educación Ambiental y II Congreso Nacional de Investigación en la Enseñanza de la Biología

Jiménez, M.P. (1991). Cambiando las ideas sobre el cambio biológico. Enseñanza de las Ciencias, 9(3), 248- 256.

Jiménez, M. P. (1994). Teaching evolution and natural selection: a look at textbooks and teachers. Journal of Research in Science Teaching, 31(5), 519- 536.

Maciel, S. (2005). Concepciones sobre la evolución biológica de estudiantes de licenciatura en educación primaria de la Benemérita Escuela Nacional de Maestros. (Tesis de Pregrado). Facultad de Ciencias, UNAM.

Maciel, S. (2007). Concepciones sobre evolución biológica. presentes en estudiantes de licenciatura en educación primaria. IX Congreso Nacional de Investigación Educativa. Mérida, México.

Recuperada

en: http://www.comie.org.mx/congreso/memoria/v9/ponencias/at05/PRE1178331160.p df

Maldonado-Rivera, J. (1998). An examination of the factors affecting the teaching and learning of evolution. (Tesis doctoral). Columbia University. Recupeda de la base de datos Dissertation Abstracts International, 59-07, Section: A, 2431.

Mayr, E. (2000). Darwin`s Influence on the modern Thougth. American Scientific, julio: $79-83$.

Miller, J., Scott, E. y Okamoto, S. (2006). Public Acceptance of Evolution. Science, 313(5788), 765-766.

Moore R. y Cotner S. (2009). Educational Malpractice: The Impact of Including Creationism in High School Biology Courses. Evo Edu Outreach, 2(1), 95-100

Munger, J. 2002, Unwarranted Conclusions Regarding Attitudes About Evolution. The American Biology Teacher, 64(1), 9-10.

Nadelson, L. y Southerland, S. (2010). Examining the Interaction of Acceptance and Understanding: How Does the Relationship Change with a Focus on Macroevolution?. Evo Edu Outreach, 3(1), 82-88

Özay, E. (2010). Biology students' and teachers' religious beliefs and attitudes towards theory of evolution. H. U. Journal of Education, 38, 189-200

Paz y Miño, G. y Espinosa, A. (2009). Acceptance of Evolution Increases with Student Academic Level: A Comparison Between a Secular and a Religious 
Bio-grafia Escritos sobre la Biologia y su Enseñanza.

Edición Extra-Ordinaria. ISSN 2027-1034 P. p 666-675

Memorias del VII Encuentro Nacional de Experiencias en la Enseñanza de la Biología y la Educación Ambiental y II Congreso Nacional de Investigación en la Enseñanza de la Biología

College. Evo Edu Outreach, 2(4), 655-675.

Paz y Miño, G. y Espinosa, A. (2011). New England Faculty and College Students Differ in Their Views About Evolution, Creationism, Intelligent Design, and Religiosity. Evo Edu Outreach, 4, 323-342

Peker, D., Gul C. y Aykut, K. (2010). Three Decades of Anti-evolution Campaign and its Results: Turkish Undergraduates' Acceptance and Understanding of the Biological Evolution Theory. Science \& Education, 19(6-8), 739-755

Pigliucci, M. (2007). The evolution-creation wars: why teaching more science just is not enough. McGill Journal of Education, 42(2), 285-306.

Roelfs, F. C. (1987). Academic Factors Affecting the Status of the Teaching of Evolution in Arkansas and Missouri. (Tesis doctoral). University of MissouriColumbia, EU.

Rosenthal, D. B. (1985). Evolution in High School Biology Textbboks: 1963-1983. Science Education, 69(5), 637-648.

Scott, E. y Branch, G. (2003). Antievolutionism: Changes and continuities. Bioscience, 53(3), 282-285.

Shankar, G. (1990). Factors influencing the teaching of evolution and creationism in Texas public high school biology classes. (Tesis doctoral). Texas Tech University. Recuperada de la base de datos Dissertation Abstracts International 51:03733A.

Skoog, G. (1978). Does Creationism Belong in the Biology Curriculum?. American Biology Teacher, 40, 23-26.

Skoog, G. (1979). Topic of Evolution in Secondary School Biology Textbooks: 19001977. Science Education, 63(5), 621-640.

Skoog, G. (1984). The Coverage of Evolution in High School Biology Textbooks Published in the 1980s. Science Education, 68(2), 117-128.

Soto-sonera, J. (2006). Implicaciones educativas de las creencias religiosas de las docentes de ciencia en la enseñanza del tema "evolución biológica": Un estudio de caso. Revista Paideia Puertorriqueña, Universidad de Puerto Rico, 1(2).

Recuperado de: http://paideia.uprrp.edu

Soto-sonera, J. (2009). Influencia de las creencias religiosas en los docentes de 
Bio-grafia Escritos sobre la Biologia y su Enseñanza.

Edición Extra-Ordinaria. ISSN 2027-1034 P.p 666 - 675

Memorias del VII Encuentro Nacional de Experiencias en la Enseñanza de la Biologia y la Educación Ambiental y II Congreso Nacional de Investigación en la Enseñanza de La Biología

ciencia sobre la teoría de la evolución biológica y su didáctica. Revista Mexicana de Investigación Educativa, 14(41), 515-538.

Southcott, R. \& Downie, R. (2012). Evolution and Religion: Attitudes of Scottish Bioscience Students to the Teaching of Evolutionary Biology. Evo Edu Outreach, 5, 301-311. 